\title{
Emergencia y características agronómicas del Cucumis sativus a la aplicación de quitosano, Glomus cubense y ácidos húmicos
}

\author{
Emergency and agronomic characteristics of Cucumis sativus after the application of chitosan, \\ Glomus cubense and humic acids
}

\begin{abstract}
Juan José Reyes-Pérez', Marisol Rivero-Herrada', Cristian Josué Andagoya Fajardo', Félix Alfredo Beltrán-Morales², Luís Guillermo Hernández-Montiel ${ }^{3}$, Ana Elizabeth García Liscano ${ }^{4}$ y Francisco Higinio Ruiz-Espinoza ${ }^{2 *}$

1 Universidad Técnica Estatal de Quevedo. Av. Quito. Km 1 1⁄2 vía a Santo Domingo. Quevedo, Los Ríos, Ecuador.

2 Universidad Autónoma de Baja California Sur, La Paz, Baja California Sur, México.

3 Centro de Investigaciones Biológicas del Noroeste, S.C. Mar Bermejo No. 195, Col. Playa Palo de Santa Rita, La Paz, Baja California Sur 23090, México.

4 Instituto Tecnológico Superior Ciudad de Valencia, Quevedo, Ecuador.
\end{abstract}

\section{RESUMEN}

El objetivo de la investigación fue evaluar el comportamiento de la emergencia y características agronómicas del cultivo de pepino (Cucumis sativus L.) ante la aplicación de quitosano, hongos micorrízicos y ácidos húmicos en condiciones protegidas. La investigación se realizó en un invernadero y laboratorio de Biología Molecular y Microbiología de la Universidad Técnica Estatal de Quevedo, Ecuador. El material vegetativo fueron las variedades comerciales de pepino (Inivit y Racer), con tratamientos de quitosano $\left(3 \mathrm{~g} \mathrm{~L}^{-1}\right)$, Glomus cubense (20 esporas/100 g de suelo) y ácidos húmicos (1:30 v/v). Se evaluó emergencia, variables morfométricas, indicadores de crecimiento e indicadores de producción. Los resultados indicaron que la variedad Racer registró el mayor porcentaje de emergencia, el hongo Glomus cubense presentó la mayor emergencia (71.7\%). Mientras el Glomus cubense en la variedad Inivit mostró la mayor biomasa fresca (22.6 g). En tanto que el quitosano ( $285.8 \mathrm{~g}$ ) y el Glomus cubense (277.9 g) influyeron en el peso del fruto. A su vez el quitosano en la variedad Inivit generó el mayor rendimiento $\left(5,703 \mathrm{~kg} \mathrm{ha}^{-1}\right)$. En general se comprobó que la aplicación del hongo Glomus cubense induce a incrementos en la emergencia, biomasa fresca, biomasa seca del cultivo de pepino, por su parte la aplicación de quitosano en ambas variedades generó mayor rendimiento.

Palabras claves: Bioestimulantes, biofertilización, producción, germinación, biomasa.

\section{ABSTRACT}

The objective of the research was to evaluate the emergence behavior and agronomic characteristics of the cucumber (Cucumis sativus L.) crop with the application of chitosan, mycorrhizal fungi and humic acids under protected conditions. The research was carried out in a greenhouse and a laboratory of Molecular Biology and Microbiology of the State Technical University of Quevedo, Ecuador. The vegetative material was the commercial varieties of cucumber (Inivit and Racer), with chitosan (3 $\left.\mathrm{g} \mathrm{L}^{-1}\right)$, Glomus cubense fungi (20

Volumen XXIII, Número 3 spores/100 $\mathrm{g}$ of soil) and humic acids (1:30 v/v) treatments. Emergence, morphometric variables, growth indicators and production indicators were evaluated. The results indicated that the Racer variety registered the highest percentage of emergence, and the Glomus cubense fungi presented the highest emergence (71.7\%). On the other hand, the Glomus cubense fungi showed the highest fresh biomass $(22.6 \mathrm{~g})$ in the Inivit variety. Chitosan $(285.8 \mathrm{~g})$ and the Glomus cubense fungi $(277.9 \mathrm{~g})$ influenced fruit weight. Chitosan in the Inivit variety $(5703.7 \mathrm{~g})$ generated the highest yield. In general, it was found that the application of the Glomus cubense fungi induces an increase in emergence, fresh biomass, dry biomass of the cucumber crop, while the application of chitosan in both varieties generated higher yields.

Keywords: Biostimulants, biofertilization, production, germination, biomass.

\section{INTRODUCCIÓN}

El pepino (Cucumis sativus L.), es una planta cultivada en casi todo el mundo y cuyo fruto tiene bastante aceptación entre la población, originario de la India y domesticado en Asia (López et al,. 2011; Reyes et al., 2017). Las variedades más comunes del pepino son el americano, el europeo, el holandés y el pepino oriental (Leiva, 2018). Por lo que es uno de los cultivos con más demanda mundial, al ser muy utilizado en la dieta del ser humano, ya que al consumir un pepino equivale a beberse un vaso de agua, debido a el $96 \%$ de su peso es agua y el $4 \%$ restante es materia sólida con un gran valor nutritivo, pues es una gran fuente de minerales y vitaminas (Flores, 2017).

En el Ecuador el pepino es una hortaliza cultivada principalmente por pequeños grupos de productores en áreas reducidas de terreno agrícola, que, aun así, asumen la responsabilidad de mantener disponibles esta hortaliza en los mercados nacionales, sin embargo, el problema, es no poseer alternativas agroecológicas que se introduzcan en el proceso de producción y que sean accesible para los mismos. Según el Sistema de Información Pública Agropecuaria (2018), actualmente el área de siembra en Ecuador, se ha

*Autores para correspondencia: Francisco Higinio Ruiz-Espinoza Correo electrónico: fruiz@uabcs.mx

Recibido: 26 de enero de 2021

Aceptado: 26 de marzo de 2021 
incrementado desde el año 2010, de tal manera que existen aproximadamente 1,842 ha cosechadas.

A nivel mundial y en el Ecuador la agricultura, que entre sus procesos productivos requiere del uso de insumos diferentes a los comúnmente utilizados como los fertilizantes químicos, por lo que el aumento de productos que tiendan a reducir el impacto ambiental y que cada vez se vuelvan más rentables, esto debido a la eficiencia y accesibilidad a otras alternativas, entre las que figuran el uso de bioestimulantes como el quitosano, hongos micorrízicos arbusculares (HMA) y ácidos húmicos (Torres et al., 2018; Baker et al., 2020).

Durante la última década los estudios enfocados a la aplicabilidad del quitosano en otras áreas como la agricultura se han incrementado debido principalmente al mejoramiento del crecimiento y productividad y calidad de frutos de diferentes cultivos (Domini, 2018, Basit et al., 2020).

Por otra parte, el uso de los ácidos húmicos en plantas, están relacionados con la pontencialización de la fisiología de las plantas, promover el crecimiento y mejorar la respuesta al estrés (Veobides et al., 2018), formación de raíces adventicias (Baldotto et al., 2012), elongación de la raíz y la formación de pelos de la raíz (Canellas et al. 2015). Todos estos factores incrementan el área de la masa de raíces (volumen de raíces) y la superficie de las raíces, lo que contribuye a una mayor absorción de agua y nutrientes (Eyheraguibel et al., 2008).

El uso de las micorrizas arbusculares mejoran el crecimiento vegetal (Medina, 2016), en el mismo sentido Arias et al. (2019) mencionan que la colonización micorrízica produce cambios físicos, bioquímicos y fisiológicos en las raíces que conducen a un mejor estado general de la planta y contribuyen a aliviar las situaciones de estrés de carácter abiótico (metales pesados, salinidad) y bióticos (ataques de patógenos, cambios microbianos en la rizosfera) (Moreno et al., 2018). Por estos motivos mencionados anteriormente y la necesidad de buscar opciones diferentes, y con beneficio a la salud humana, ambiental, social, y nuevas tecnologías de producción agrícola, se desarrolló una investigación donde el objetivo fue evaluar el comportamiento de la emergencia y características agronómicas del cultivo de pepino (Cucumis sativus L.) ante la aplicación de quitosano, hongos micorrízicos (Glomus cubense) y ácidos húmicos en condiciones protegidas

\section{MATERIALES Y MÉTODOS}

El desarrollo de la investigación se llevó a cabo en el invernadero del Campus Experimental "La María" ubicado en el km 7.5 de la vía Quevedo-Mocache en la zona del cantón Mocache, provincia de Los Ríos, ubicada en el kilómetro 7.1/2 de la vía Quevedo - El Empalme. La ubicación geográfica es $1^{\circ} 04^{\prime} 48.6^{\prime \prime}$ latitud Sur y $79^{\circ} 30^{\prime} 04.2^{\prime \prime}$ longitud Oeste, a una altitud de $75 \mathrm{~m}$ sobre el nivel del mar. El predio se encuentra en una zona climática tropical húmeda, con temperatura media anual de $24,8^{\circ} \mathrm{C}$, precipitación media anual de $2252 \mathrm{~mm} ; 84$ $\%$ de humedad relativa y $894.0 \mathrm{~h}$ de sol al año. Los cultivares estudiados fueron: Inivit y Racer. Las semillas se desinfectaron con $\mathrm{Ca}(\mathrm{ClO})_{2}$ al $5 \%$, imbibiéndose por $5 \mathrm{~min}$, lavado (agua destilada), dejándose en reposo por 4 horas sin agua.

\section{Diseño experimental}

Se evaluaron 6 tratamientos (Tabla 1), donde se incluyó un testigo de cada variedad con agua destilada, el diseño experimental utilizado fue un completamente al azar con arreglo factorial $2 \times 4$ en 3 repeticiones, siendo el primer factor las variedades y el segundo factor los bioestimulantes, con su respectivo control.

Tabla 1. Tratamientos del uso de Quitosano, Glomus cubense y ácidos húmicos en pepino.

Table 1. Treatments for the use of Chitosan, Glomus cubense and humic acids in cucumber.

\begin{tabular}{|c|c|c|}
\hline $\begin{array}{c}\text { Factor A } \\
\text { Variedades } \\
\text { pepino }\end{array}$ & $\begin{array}{c}\text { Factor B } \\
\text { (Bioestimulantes) }\end{array}$ & Tratamientos \\
\hline \multirow{8}{*}{ V1: Inivit } & & V1T1: Inivit + Quitosano \\
\hline & T1. Ouitosano & $\begin{array}{c}\text { V1T2: Inivit + Glomus } \\
\text { cubense }\end{array}$ \\
\hline & ( $3 \mathrm{~g} \mathrm{~L}^{-1}$ de agua) & $\begin{array}{l}\text { V1T3: Inivit + Ácidos } \\
\text { húmicos }\end{array}$ \\
\hline & $\begin{array}{c}\text { T2: Glomus cubense } \\
\text { (20 esporas/100 g de suelo) }\end{array}$ & V1T4: Inivit + Agua \\
\hline & & V2T1: Racer + Quitosano \\
\hline & $\begin{array}{l}\text { 1:: Acidos numicos } \\
(1: \mathrm{v})\end{array}$ & $\begin{array}{c}\text { V2T2: } \text { Racer + Glomus } \\
\text { cubense }\end{array}$ \\
\hline & \multirow[t]{2}{*}{ T4: Control } & $\begin{array}{l}\text { V2T3: } \text { Racer + Ácidos } \\
\text { húmicos }\end{array}$ \\
\hline & & V2T4: Racer + Agua \\
\hline
\end{tabular}

\section{Desarrollo del experimento}

El trabajo se llevó a cabo en invernadero casa sombra con una superficie de $20 \mathrm{~m}$ de largo $\times 6 \mathrm{~m}$ de ancho con una superficie total de $120 \mathrm{~m}^{2}$, primeramente se preparó el sustrato para el semillero, con una mezcla de 4:2:1, cuatro partes de tierra, dos partes de materia orgánica obtenida de vermicomposta y una parte de cascarilla de arroz, posteriormente, se realizaron los tratamientos de las semillas, estas imbibieron de los bioestimulantes por un lapso de 10 minutos, para luego dejarse secar por un período de 4 horas antes de la siembra. La siembra se realizó de forma directa en bolsas de plástico de $60 \times 60 \mathrm{~cm}$, con una densidad de 10 macetas por $\mathrm{m}^{2}$.

\section{Aplicación de los tratamientos}

La aplicación del quitosano $3 \mathrm{~g} \mathrm{~L}^{-1}$ se realizó de acuerdo a lo propuesto por González et al. (2015), mientras que los hongos micorrízicos arbusculares se empleó la especie Glomus cubense en dosis de 20 esporas/100 g suelo según la metodología de Rivera et al. (2003), mientras que los ácidos húmicos fueron 1:30 v/v de acuerdo a Chen et al. (2004), la primera aplicación se realizó a los 7 días después de la siembra, posteriormente se realizaron 3 aplicaciones más después de la primera en intervalos de 7 días.

\section{Manejo del cultivo}

El tutorado se realizó a los 20 después de la siembra, se realizó una poda a los 30 días después de la siembra, eliminando los brotes axilares, dejando los ejes productivos, 
seguidamente se aplicó oxicloruro de cobre $\mathrm{Cu}_{2}(\mathrm{OH})_{3} \mathrm{Cl}(3 \mathrm{~g}$ $\mathrm{L}^{-1}$ de agua) en aspersión foliar. Cuando se presentaron insectos plaga se efectuaron dos aplicaciones (7 y 15 después de la siembra), de Actara (IA: Thiamethoxam) en dosis de $0.3 \mathrm{~kg}$ $\mathrm{ha}^{-1}$, mientas que para el control de enfermedades se aplicó Daconil (IA: Clorotalonil) en dosis de $1.50 \mathrm{~L} \mathrm{ha}^{-1}$. Por su parte la cosecha se realizó a los 60 días después de la siembra al alcanzar la madurez comercial.

\section{Variables estudiadas}

Las variables evaluadas fueron: Porcentaje de emergencia (E) de acuerdo a Maguire (1962) , , la biomasa fresca y seca considero la planta completa (tallos, hojas y raíces), esta se determinó al pesar cada plántula, en una balanza analítica expresándose en gramos de materia vegetal fresca por su parte la biomasa seca se determinó al colocar la biomasa fresca en una estufa de secado, a temperatura de 80 ${ }^{\circ} \mathrm{C}$ durante $72 \mathrm{~h}$. En cuanto a los indicadores de crecimiento evaluados fueron: Altura de planta $(\mathrm{cm})$, se consideraron 10 plantas por cada unidad experimental a los 35 después de siembra, diámetro de tallo $(\mathrm{mm})$ igualmente a los a los 35 después de siembra mediante un calibrador de vernier, el número de hojas a los 45 después de siembra, en cuanto al número de flores por planta se realizó cuando la floración fue al $50 \%$, esto ocurrió entre los 40 y 45 después de siembra. En lo referente a los indicadores de producción, fueron el número de frutos por planta, se contaron por planta de cada tratamiento. Previamente para el desarrollo de la investigación se estableció el registrar esta variable solo con datos obtenidos de la primera cosecha, en cuanto a masa fresca del fruto $(\mathrm{g})$, se procedió al registro de la masa en balanza analítica, por último, el rendimiento $\left(\mathrm{kg} \mathrm{ha}^{-1}\right)$, se obtuvo al pesar los frutos cosechados por cada tratamiento, para posteriormente extrapolar a kg ha-1.

\section{Procesamiento estadístico}

Los datos se procesaron a través del Paquete Estadístico Statistica v. 10.0 empleando un análisis de varianza de clasificación simple. En el caso de los datos en porcentaje fueron trasformados mediante la fórmula Arc Sen $\sqrt{ }$ x. Een los casos en que las variables mostraron diferencias estadísticas significativas, se utilizó la prueba de comparación múltiple de medias de Tukey $(p \leq 0,05)$.

\section{RESULTADOS Y DISCUSIÓN Emergencia}

En la emergencia, el quitosano (T1) fue similar a los ácidos húmicos (T3), mientras que el Glomus cubense (Tabla 2), jugó un papel importante en la emergencia, la variedad Racer (V2) logró el mayor porcentaje de emergencia superando a la variedad Inivit (V1) en $8.9 \%(p \leq 0,05)$. En lo referente a los bioestimulantes el porcentaje de mayor emergencia, se mostró con la aplicación del Glomus cubense (T2) (Tabla 2), pero en igualdad estadística con el control (T4) y los ácidos húmicos (T3), sin embargo mientras que las interacciones entre variedades y bioestimulantes, todos los tratamientos
Tabla 2. Porcentaje de emergencia en variedades de pepino a la aplicación de Quitosano, Glomus cubense y ácidos húmicos en condiciones protegidas. Table 2. Germination emergence in cucumber varieties after the application of Chitosan, Glomus cubense and humic acids under protected conditions.

\begin{tabular}{lcc}
\hline Tratamientos & \multicolumn{2}{c}{ PE } \\
\hline Variedades & 57.5 & $\mathrm{~b}$ \\
\hline V1: Inivit & 66.4 & $\mathrm{a}$ \\
\hline V2: Racer & & \\
\hline Tratamientos & 48.4 & $\mathrm{~b}$ \\
\hline T1: Quitosano & 71.7 & $\mathrm{a}$ \\
\hline T2: Glomus cubense & 60.5 & $\mathrm{a}$ \\
\hline T3: Ácidos húmicos & 67.2 & $\mathrm{a}$ \\
\hline T4: Agua & & \\
\hline Interacciones & 30.0 & $\mathrm{~b}$ \\
\hline V1T1: Inivit + Quitosano & 73.3 & $\mathrm{a}$ \\
\hline V1T2: Inivit + Glomus cubense & 52.2 & $\mathrm{a}$ \\
\hline V1T3: Inivit + Ácidos húmicos & 74.4 & $\mathrm{a}$ \\
\hline V1T4: Inivit + Agua & 66.7 & $\mathrm{a}$ \\
\hline V2T1: Racer + Quitosano & 70.0 & $\mathrm{a}$ \\
\hline V2T2: Racer + Glomus cubense & 68.9 & $\mathrm{a}$ \\
\hline V2T3: Racer + Ácidos húmicos & 60.0 & $\mathrm{a}$ \\
\hline V2T4: Racer + Agua & 61.9 & \\
\hline Promedio & 13.6 & \\
\hline Coeficiente de variación (\%) & & \\
\hline PE:Porcenta de & & \\
\hline
\end{tabular}

PE: Porcentaje de emergencia. T1: $3 \mathrm{~g} \mathrm{~L}^{-1}$ de agua; T2: 20 esporas/100 $\mathrm{g}$ de suelo; T3: $3 \mathrm{~g} \mathrm{~L}^{-1}$ de agua; T4: Agua destilada. * Promedios con la misma letra en cada grupo de datos no difieren estadísticamente según la prueba de Tukey $\mathrm{P} \leq 0.05$

fueron estadísticamente iguales presentando valores que fluctúan entre 73.3 y $52.2 \%$, a excepción del tratamiento de la variedad Inivit con la aplicación de Quitosano (V1T1) que presentó el menor porcentaje de emergencia.

Los resultados encontrados entre variedades podrían explicarse debido a que posiblemente se tenga diferencias de reserva energética para la germinación embrionaria y no directamente por la viabilidad de los embriones, debido a la heterogeneidad en la madurez de la semilla (Doria, 2010). En este mismo sentido Ellis y Roberts (1980) indican que, efectivamente la viabilidad también depende de las variaciones genéticas dentro de una misma especie y de las condiciones previas al almacenamiento. Lo cual concuerda plenamente con los resultados encontrados en el presente estudio, dado que mientras la variedad Inivit presentó menor emergencia que la variedad Racer, estos resultados concuerdan con lo reportado por Ramírez et al. (2018), donde mencionan que con HMA se ve influida la germinación y emergencia de plántulas de arboles en vívero. Por su parte Vital et al. (2018) en semillas de girasol con HMA, señalan que la germinación de estas semillas se ve influenciada por el genotipo y el tiempo de almacenamiento. En este sentido Flores et al (2020), afirman que la expresión de diferentes respuestas de las semillas a la 
colonización de HMA es determinada por el carácter genotípico del huésped y del hospedero.

\section{Biomasa fresca y biomasa seca}

En los promedios de biomasa fresca de planta, el análisis de varianza $(\mathrm{p} \leq 0.05)$ determinó, que tanto, variedades, tratamientos e interacciones entre bioestimulantes y variedades presentaron significancia (Tabla 3). El mayor peso fresco de planta se obtuvo en la variedad Inivit (V1) superando a la variedad Racer (V2), mientras en la aplicación de los bioestimulantes, el Glomus cubense (T2) presentó el mayor valor, pero estadísticamente similar a los ácidos húmicos (T3) y al control, donde la aplicación con Quitosano (T1) registró el menor valor (Tabla 3).

En cuanto a la interacción entre variedades y bioestimulantes en la variable biomasa fresca el Glomus cubense en la variedad Inivit (V1T2) registró la mayor biomasa fresca por planta, sin embargo este tratamiento fue similar estadísticamente a los V1T3 y V1T4 (control), donde la aplicación del bioestimulante quitosano en la variedad Racer (V2T1) registró el menor valor.

La variable biomasa seca de planta, presentó diferencias estadísticas $(p \leq 0.05)$ entre los tratamientos e interacciones tratamientos con variedades, entre variedades no se

Tabla 3. Biomasa fresca y seca en variedades de pepino a la aplicación de Quitosano, Glomus cubense y ácidos húmicos en condiciones protegidas. Table 3. Fresh and dry biomass in cucumber varieties after the application of Chitosan, Glomus cubense and humic acids under protected conditions.

\begin{tabular}{|c|c|c|}
\hline Tratamientos & $B F(g)$ & BS (g) \\
\hline \multicolumn{3}{|l|}{ Variedades } \\
\hline V1: Inivit & $17.7 \mathrm{a}$ & $7.00 \mathrm{a}$ \\
\hline V2: Racer & $14.2 \mathrm{~b}$ & $6.70 \mathrm{a}$ \\
\hline \multicolumn{3}{|l|}{ Tratamientos } \\
\hline T1: Quitosano & $13.43 \mathrm{~b}$ & $6.30 \mathrm{ab}$ \\
\hline T2: Glomus cubense & $18.17 \mathrm{a}$ & $8.40 \mathrm{a}$ \\
\hline T3: Ácidos húmicos & $15.57 \mathrm{ab}$ & $5.30 \mathrm{~b}$ \\
\hline T4: Agua & $16.53 \mathrm{ab}$ & $7.60 \mathrm{ab}$ \\
\hline \multicolumn{3}{|l|}{ Interacciones } \\
\hline V1T1: Inivit + Quitosano & $13.5 \mathrm{~b}$ & $5.80 \mathrm{a} \mathrm{b}$ \\
\hline V1T2: Inivit + Glomus cubense & $22.6 \mathrm{a}$ & $9.33 \mathrm{a}$ \\
\hline V1T3: Inivit + Ácidos húmicos & $16.1 \mathrm{a} \quad \mathrm{b}$ & $5.50 \mathrm{a} \mathrm{b}$ \\
\hline V1T4: Inivit + Agua & $18.4 \mathrm{a} \quad \mathrm{b}$ & $7.50 \mathrm{a} \mathrm{b}$ \\
\hline V2T1: Racer + Quitosano & $13.4 \quad b$ & $6.70 \mathrm{a} \mathrm{b}$ \\
\hline V2T2: Racer + Glomus cubense & 13.7 & $7.43 \mathrm{a} \mathrm{b}$ \\
\hline V2T3: Racer + Ácidos húmicos & 15.0 & $5.13 \mathrm{~b}$ \\
\hline V2T4: Racer + Agua & 14.6 & $7.70 \mathrm{a} \mathrm{b}$ \\
\hline Promedio & 15.9 & 6.80 \\
\hline Coeficiente de variación (\%) & 15.04 & 20.26 \\
\hline
\end{tabular}

BF: Biomasa fresca, BS: Biomasa seca. T1: $3 \mathrm{~g} \mathrm{~L}^{-1}$ de agua; T2: 20 esporas $/ 100$ $\mathrm{g}$ de suelo; T3: $3 \mathrm{~g} \mathrm{~L}^{-1}$ de agua; T4: Agua destilada. *Promedios con la misma letra en cada grupo de datos no difieren estadísticamente según la prueba de Tukey $\mathrm{P} \leq 0.05$. presentó diferencias, sin embargo númericamente la variedad Inivit (V1) mostró mayor biomasa seca que la variedad Racer (V2), posiblemente debido el genotipo y el tiempo de almacenamiento. En lo que concierne a los bioestimulantes el T2 (Glomus cubense) presentó mayor biomasa seca, pero sin diferencias estadísticas con T1 y T4, siendo la aplicación con ácidos húmicos (T3) la que registró el valor menor. Por su parte las interacciones entre variedades y bioestimulantes, el V1T2 a base de Glomus cubense en la variedad Inivit registró la mayor biomasa seca de planta, mientras las demás interacciones que presentaron valores que oscilan entre $7,7 \mathrm{~g}$ a 5,5 g fueron similares estadísticamente, mientras que el tratamiento V2T3 (ácidos húmicos en la variedad Racer) mostró el menor promedio de biomasa seca (Tabla 3).

El efecto de los bioestimulantes quitosano, el Glomus cubense y ácidos húmicos, como promotores de crecimiento vegetal en pepino fueron estadísticamente diferentes ( $p \leq$ 0.05 ) en la mayoría de las variables de crecimiento evaluadas; con respecto a la variable altura, los promedios en el análisis de varianza arrojó (Tabla 4), que en las variedades, tratamientos e interacciones variedades con bioestimulantes no presentaron significancia $(p \leq 0.05)$.

Tabla 4. Altura, diámetro del tallo, biomasa fresca, número de hojas y número de flores en variedades de pepino a la aplicación de Quitosano, Glomus cubense y ácidos húmicos en condiciones protegidas.

Table 4. Height, stem diameter, fresh biomass, number of leaves and number of flowers in cucumber varieties after the application of Chitosan, Glomus cubense and humic acids under protected conditions.

\begin{tabular}{|c|c|c|c|c|}
\hline Tratamientos & $A(\mathrm{~cm})$ & DT (mm) & NH & $\mathbf{N F}$ \\
\hline \multicolumn{5}{|l|}{ Variedades } \\
\hline V1: Inivit & $25.3 \mathrm{a}$ & $4.5 \mathrm{a}$ & $9.3 \mathrm{a}$ & $7.0 \mathrm{a}$ \\
\hline V2: Racer & $24.3 \mathrm{a}$ & $4.1 \mathrm{~b}$ & $7.5 \mathrm{~b}$ & $6.5 \mathrm{a}$ \\
\hline \multicolumn{5}{|l|}{ Tratamientos } \\
\hline T1: Quitosano & $25.2 \mathrm{a}$ & $4.4 \mathrm{a}$ & $8.8 \mathrm{a}$ & $6.7 \mathrm{a}$ \\
\hline T2: Glomus cubense & $24.8 \mathrm{a}$ & $4.6 \mathrm{a}$ & $7.7 \mathrm{~b}$ & $6.5 \mathrm{a}$ \\
\hline T3: Ácidos húmicos & $25.1 \mathrm{a}$ & $4.5 \mathrm{a}$ & $8.3 \mathrm{ab}$ & $7.0 \mathrm{a}$ \\
\hline T4: Agua & $24.2 \mathrm{a}$ & $3.7 \mathrm{~b}$ & $8.7 \mathrm{a}$ & $7.0 \mathrm{a}$ \\
\hline \multicolumn{5}{|l|}{ Interacciones } \\
\hline V1T1: Inivit + Quitosano & $27.4 \mathrm{a}$ & $4.8 \mathrm{a}$ & $10.1 \mathrm{a}$ & $7.4 \mathrm{a}$ \\
\hline V1T2: Inivit + Glomus cubense & $24.2 \mathrm{a}$ & $4.7 \mathrm{a}$ & $7.7 \mathrm{bc}$ & $7.1 \mathrm{a}$ \\
\hline V1T3: Inivit + Ácidos húmicos & $25.0 \mathrm{a}$ & $4.7 \mathrm{ab}$ & $9.2 \mathrm{a}$ & $6.7 \mathrm{a}$ \\
\hline V1T4: Inivit + Agua & $24.5 \mathrm{a}$ & $3.7 \mathrm{bc}$ & $10.2 \mathrm{a}$ & $7.0 \mathrm{a}$ \\
\hline V2T1: Racer + Quitosano & $22.9 a$ & $4.1 \mathrm{abc}$ & $7.5 \mathrm{~b}$ & $6.0 \mathrm{a}$ \\
\hline V2T2: Racer + Glomus cubense & $25.4 \mathrm{a}$ & $4.4 \mathrm{abc}$ & $7.7 \mathrm{~b}$ & $5.9 \mathrm{a}$ \\
\hline V2T3: Racer + Ácidos húmicos & $25.1 \mathrm{a}$ & $4.2 \mathrm{abc}$ & $7.4 \mathrm{~b}$ & $7.3 \mathrm{a}$ \\
\hline V2T4: Racer + Agua & $23.8 \mathrm{a}$ & 3.7 & $7.3 \mathrm{~b}$ & $7.0 \mathrm{a}$ \\
\hline Promedio & 24.8 & 4.3 & 8.4 & 6.8 \\
\hline Coeficiente de variación (\%) & 11.18 & 7.8 & 5.3 & 13.1 \\
\hline
\end{tabular}

A: Altura de planta, DT: Diámetro del tallo, NH: Número de hojas por planta, NF: Número de flores por planta

(cm). T1: $3 \mathrm{~g} \mathrm{~L}^{-1}$ de agua; T2: 20 esporas/100 $\mathrm{g}$ de suelo; T3: $3 \mathrm{~g} \mathrm{~L}^{-1}$ de agua; T4: Agua destilada. *Promedios con la misma letra en cada grupo de datos no difieren estadísticamente según la prueba de Tukey $p \leq 0.05$. 


\section{Diámetro del tallo y Número de hojas}

La variable diámetro de tallo en la variedad Inivit (V1) mostró ser diferente a la variedad Racer (V2). En cuanto a las aplicaciones de los bioestimulantes con Quitosano (T1), el Glomus cubense (T2) y ácidos húmicos (T3) fueron estadísticamente iguales $(p \leq 0.05)$, pero superior al tratamiento control (T4). En lo que concierne a las interacciones variedadbioestimulantes, los tratamientos V1T1 (variedad Inivit con la aplicación de Quitosano) y el tratamiento V1T2 (Inivit con Glomus cubense) registraron los mayores promedios de diámetro de tallo, siendo superiores a las demás interacciones, la menor respuesta al diámetro se presentó en la variedad Racer con el tratamiento control (V2T4).

La variable número de hojas (Tabla 4) presentó diferencias $(p \leq 0.05)$ entre variedades, tratamientos e interacciones bioestimulantes-variedad. La variedad Inivit (T1) mostró el mayor número de hojas por planta, superando a la variedad Racer (V2). Por su parte el Quitosano (T1) y control (T4) fueron los que registraron los mayores promedios por planta, donde la aplicación del Glomus cubense quien registró el menor promedio con 7.7 hojas. Las interacciones de la variedad Inivit con la aplicación de Quitosano (V1T1), ácidos húmicos (V1T3) y tratamiento control (V1T4) registraron los mayores promedios.

El uso de bioestimulantes en los procesos de producción agrícola va en aumento, dado a sus beneficios que aportan mediante hormonas vegetales como giberelinas, auxinas, citocininas, compuestos, y/o microorganismos, cuyo uso funcional va desde mejorar el metabolismo en el crecimiento y desarrollo de las plantas (Brown y Saa, 2015). Según Moreno (2018) cuando se aplican los HMA a las plantas o la rizosfera, implica la mejoría del desarrollo del cultivo, vigor, rendimiento y/o la calidad mediante la estimulación de procesos naturales que benefician el crecimiento y las respuestas a estrés biótico y/o abiótico.

Las respuesta positiva del Glomus cubense se hicieron presentes en el peso de la biomasa fresca de la planta, en donde el Glomus cubense en la variedad Inivit registró el mayor peso fresco de planta, esto según Medina (2016), se da porque la colonización micorrízica produce cambios físicos, bioquímicos y fisiológicos en las raíces que conducen a un mejor estado general de la planta, y por ende da como resultado una mayor biomasa en las plantas con las que forman simbiosis. En este mismo sentido Barrer (2009) y Calderón (2013), establecen que el uso de HMA en la agricultura contribuye a mejorar el nivel nutricional de la planta lo que se ve reflejado en mayor masa seca, crecimiento y área foliar de la planta.

Por su parte Monirul et al. (2018), reportan que con la aplicación de quitosano en tomate incrementa el crecimiento del cultivo, además mayores números de flores, resultados similares a lo encontrado en nuestro trabajo. De la misma manera Shehata et al. (2012), reportaron que la aplicación de quitosano foliar incrementó el crecimiento vegetativo y el rendimiento de pepino. Asi mismo Monirul et al. (2018) reportan que la aplicación de quitosano en chile incrementó las caracteristicas morfológicas como altura, diámetro del tallo entre otras. Esto es similar a los resultados obtenidos de nuestra investigación demuestran que el quitosano posee beneficios en el cultivo del pepino en las características morfológicas y rendimiento, tal como lo menciona Pichyangkura y Chadchawan (2015), donde mencionan que este se utiliza como bioestimulante, tanto para estimular el crecimiento de las plantas, como para inducir tolerancia al estrés abiótico o resistencia a patógenos. En este mismo sentido Muscolo et al. (2013) y Canellas et al. (2015), mencionan que los ácidos húmicos exhiben efectos estimulantes en el crecimiento, el metabolismo y desarrollo de células vegetales en cualquier tipo de planta, generalmente debido a la absorción de las células vegetales superiores por su intervención en la aceleración de nutrientes.

En este sentido Araujo et al. (2012), reportaron en tabaco resultados similares a los nuestros, donde no se encontraron diferencias entre quitosano y el control en el número de hojas. Sin embargo, los resultados de nuestro trabajo son similares a los presentados por Mujica y Fuentes (2012), donde mencionan que la influencia de la inoculación de los HMA en los indicadores del desarrollo vegetativo del cultivo del tomate (altura, biomasa seca aérea) se incrementaron. En ambos casos se pudo comprobar que los tratamientos inoculados superaron al testigo, ya sea en forma líquida o sólida, sin embargo difiere en el número de flores de nuestra investigación, donde Mujica y Fuentes (2012) encontraron que la aplicación de los hongos micorrízicos indujeron la estimulación del número de flores, mientras que nuestra investigación no presentó diferencias.

Los resultados de la variable número de frutos por planta presentaron diferencias significativas $(p \leq 0.05)$ en las variedades, donde la variedad Inivit (V1) superó a la variedad Racer (V2). Mientras que la aplicación de los bioestimulates no presentó significancia, sin embargo, quitosano (T1) fue superior númericamente con 1,7 frutos por planta (Tabla 5).

En lo que respecta a las interacciones variedades $y$ bioestimulantes el tratamiento V1T1 (variedad Inivit con quitosano) presentó los mayores promedios de número de frutos por planta, sin embargo sin diferencias entre los demás los bioestimulantes.

En este sentido la variable peso del fruto en las variedades presentó diferencias ( $p \leq 0.05)$, siendo la variedad Inivit (V1) la de mayor peso de frutos, mientras que los bioestimulantes aplicados, el quitosano (T1) produjo frutos de mayor peso (Tabla 5), pero en igualdad estadística con el Glomus cubense (T2) y ácidos húmicos (T3), mientras que en las interacciones variedad y bioestimulantes la aplicación de quitosano en la variedad Inivit (V1T1) produjo frutos de mayor peso, pero sin diferencias entre los tratamientos $V 1 T 2$ y V1T3, el tratamiento control en la variedad Inivit (V1T4) y Racer (V2T4) presentaron los menores valores.

\section{Rendimiento y peso de frutos}

El rendimiento fue mayor para la variedad Inivit (V1), superando en $826.1 \mathrm{~kg} \mathrm{ha}^{-1}$ a la variedad Racer (V2). En lo que 
Tabla 5. Número de frutos, rendimiento y peso del fruto en variedades de pepino a la aplicación de Quitosano, Glomus cubense y ácidos húmicos en condiciones protegidas.

Table 5. Number of fruits, yield and weight of the fruit in cucumber varieties after the application of Chitosan, Glomus cubense and humic acids under protected conditions.

\begin{tabular}{|c|c|c|c|}
\hline Tratamientos & NFP & $\begin{array}{l}\text { PF } \\
\text { (g) }\end{array}$ & $\begin{array}{c}\mathbf{R} \\
\left(\mathrm{kg} \mathrm{ha}^{-1}\right)\end{array}$ \\
\hline \multicolumn{4}{|l|}{ Variedades } \\
\hline V1: Inivit & $1.7 \mathrm{~b}$ & $287.6 \mathrm{a}$ & $4751.0 \mathrm{a}$ \\
\hline V2: Racer & $1.4 \mathrm{a}$ & $257.3 \mathrm{~b}$ & $3924.9 \mathrm{~b}$ \\
\hline \multicolumn{4}{|l|}{ Tratamientos } \\
\hline T1: Quitosano & $1.7 \mathrm{a}$ & $285.8 \mathrm{a}$ & $4807.3 \mathrm{a}$ \\
\hline T2: Glomus cubense & $1.6 \mathrm{a}$ & $277.9 a$ & $4656.0 \mathrm{a}$ \\
\hline T3: Ácidos húmicos & $1.6 \mathrm{a}$ & $275.7 \mathrm{ab}$ & 4402.9 a \\
\hline T4: Agua & $1.4 \mathrm{a}$ & $250.3 \mathrm{~b}$ & $3485.6 \quad b$ \\
\hline \multicolumn{4}{|l|}{ Interacciones } \\
\hline V1T1: Inivit + Quitosano & $1.8 \mathrm{a}$ & 306.5 a & 5703.7 a \\
\hline V1T2: Inivit + Glomus cubense & $1.7 \mathrm{ab}$ & $300.6 \mathrm{ab}$ & $4949.7 \mathrm{ab}$ \\
\hline V1T3: Inivit + Ácidos húmicos & $1.7 \mathrm{ab}$ & 294.7 a b c & $4769.3 \mathrm{abc}$ \\
\hline V1T4: Inivit + Agua & $1.4 \mathrm{a}$ & $248.5 \quad d$ & $3581.2 \mathrm{bc}$ \\
\hline V2T1: Racer + Quitosano & $1.7 \mathrm{a}$ & $265.0 \mathrm{abcd}$ & $4362.2 \mathrm{abc}$ \\
\hline V2T2: Racer + Glomus cubense & $1.7 \mathrm{a}$ & $256.7 \mathrm{bcd}$ & $4036.4 \quad b c$ \\
\hline V2T3: Racer + Ácidos húmicos & $1.7 \mathrm{a}$ & $255.3 \mathrm{~cd}$ & $3911.0 \mathrm{bc}$ \\
\hline V2T4: Racer + Agua & $1.3 \mathrm{a}$ & $252.1 \mathrm{~cd}$ & 3389.9 \\
\hline Promedio & 1.6 & 272.4 & 4337.9 \\
\hline Coeficiente de variación (\%) & 11.5 & 5.9 & 9.2 \\
\hline
\end{tabular}

NFP: Número de frutos por planta, PF: Peso del fruto, R: Rendimiento; T1: $3 \mathrm{~g}$ $\mathrm{L}^{-1}$ de agua; T2: 20 esporas/100 g de suelo; T3: $3 \mathrm{~g} \mathrm{~L}^{-1}$ de agua; T4: Agua destilada. *Promedios con la misma letra en cada grupo de datos no difieren estadísticamente según la prueba de Tukey $\mathrm{P} \leq 0.05$.

respecta a la aplicación de los bioestimulantes, el quitosano (T1), el Glomus cubense (T2) y ácidos húmicos (T3) presentaron mayores rendimientos que el tratamiento control (T4). El promedio en que superaron los tratamientos al control fue de $1136.46 \mathrm{~kg} \mathrm{ha}^{-1}$ (Tabla 5). En lo referente a las interacciones el tratamiento V1T1 con la aplicación de Quitosano en la variedad Inivit registró el mayor rendimiento superando a los tratamientos V1T2, V1T3 y V2T1 con 745, 934.4 y 1341.5 $\mathrm{kg} \mathrm{ha}^{-1}$, pero sin diferir estadísticamente, el tratamiento de menor rendimiento fue V2T4 (control en variedad Racer).

En lo que se refiere a la variable peso del fruto, también se vio influenciado con la utilización de el Glomus cubense, este caso en la variedad Inivit, que obtuvo el mayor peso promedio de fruto frente a los demás promedios de las otras interacciones. La utilización de estos microorganismos resulta factible para cualquier sistema de producción agrícola debido a las funciones que realizan una vez que se asocian con las plantas; entre ellas encontramos: incremento en la absorción de nutrientes minerales y agua a partir de un aumento en el volumen de suelo explorado (Urgiles, 2019). El desarrollo de esta investigación permitió obtener resultados sobre la incidencia del quitosano, Glomus cubense y ácidos húmicos en el desarrollo del cultivo de pepino, desde la etapa de emergencia hasta la producción.
En este sentido Arias et al. (2012) mencionan que la inoculación de HMA en tomate, tuvo efecto en el rendimiento del cultivo y el número de frutos por planta, obtuvieron, que en los tratamientos inoculados los resultados fueron superiores comparados con el testigo, resultados similares a los de nuestra investigación. En el mismo sentido Sheikha y Malki (2011), mencionan que la aplicación de quitosano en frijol incremento el rendimiento y la calidad del ejote. Así mismo Sabreen et al. (2015), reportan incremento en el rendimiento y variables de crecimiento en calabaza, debido a procesos metabólicos en la interacción bioestimulante de los tratamientos a la semilla, el tipo de aplicación, y a la concentración utilizada. Por su parte Reyes et al. (2020) mencionan que el quitosano incrementa los rendimientos en tomate. En este mismo sentido Monirul et al. (2018) mencionan que con aplicación de quitosano en chile y tomate se encontró un nivel óptimo de rendimiento.

\section{CONCLUSIONES}

Las aplicaciones a base de quitosano y Glomus cubense influyeron, en el número de frutos, peso del fruto y rendimiento en ambas variedades.

La aplicación del bioestimulante a base de Glomus cubense presentaron incrementos en la emergencia, biomasa fresca, biomasa seca del cultivo de pepino.

La aplicación del bioestimulante a base de Glomus cubense en la variedad Inivit mostró el mayor peso fresco de planta a los 13 días después de la siembra, mientras que la aplicación de quitosano en la variedad Inivit generó el mayor rendimiento en pepino.

\section{AGRADECIMIENTOS}

A la Universidad Técnica Estatal de Quevedo, por el apoyo otorgado a través del Fondo Competitivo de Investigación Científica y Tecnológica (FOCICYT) $6^{\text {ta }}$ Convocatoria, a través del proyecto PFOC 6-20-2018 “Evaluación de derivados de Quitosano en la producción sostenible de hortalizas en sistema de cultivo orgánico".

\section{REFERENCIAS}

Araujo, A. L., Rodríguez, A.C., \& González, G.L.G. 2012. Efecto de la quitosana sobre el cultivo de tabaco (Nicotiana tabacum Lin.) en condiciones edafoclimáticas del municipio Guisa, Granma, Cuba. Revista UDO Agrícola. 12 (4):823-829.

Arias, M. R., Romero, F., A., Bañuelos, T. J., \& De la Cruz E. Y. 2019. Inoculación de hongos solubilizadores de fósforo y micorrizas arbusculares en plantas de jitomate. Revista Mexicana de Ciencias Agrícolas. 10(8):1747-1757.

Baker, B.P., Green, T.A., \& Loker, A.J. 2020. Biological control and integrated pest management in organic and conventional systems. Biological Control. 140: 1-9.

Baldotto, L.E., Baldotto, M.A., Soares, R.R., Martinez, H.E., \& Venegas, V.H. 2012. Adventitious rooting in cuttings of croton and hibiscus in response to indolbutyric acid and humic acid. Revista Ceres. 59(4): 476-483. 
Barrer, S. 2009. El uso de hongos micorrizicos arbusculares como una alternativa para la agricultura. Biotecnología en el Sector Agropecuario y Agroindustrial. 7(1):123-132.

Basit, A., Khan, H., Alam, M., Ullah, I., Shah, S., Zuhair, S., \& Ullah., I. 2020. Índices de calidad de la planta de tomate afectada por las condiciones de estrés hídrico y la aplicación de quitosano. Pure Appl Biol. 9: 1364-1375.

Brown, P., \& Saa, S. 2015. Biostimulants in agriculture. Frontiers in plant science. 6 (671):1-3.

Calderón, P.A., Marrero, C.Y., Martín, C.J., \& Mayo, I. 2013. La fertilidad de los suelos y su importancia en el empleo de bioproductos en la provincia de Sancti Spíritus. Cultivos Tropicales. 34(2): 16-23.

Canellas, L.P., Olivares, F.L, Aguiar, N.O., Jones, D.L., Nebbioso, A ., Mazzei, P., \& Piccolo, A. 2015. Ácidos húmicos y fúlvicos como bioestimulantes en horticultura. Sci. Hortico. 196: 15-27.

Chen Y., De Nobili M., \& Aviad T., 2004. Stimulatory effects of humic substances on plant growth. In: Soil organic matter in sustainable agriculture (Magdoff F., Weil R.R., eds). CRC Press, NY, USA.

Domini, A.K. 2018. Potencialidades del quitosano para la fresa. Usos en la mejora y conservación de los frutos. Cultivos Tropicales. 39(1): 134-142.

Doria, J. 2010. Generalidades sobre las semillas: su producción, conservación y almacenamiento. Cultivos Tropicales. 31(1):74-85.

Ellis, R.H., \& Roberts, E.H. 1980. Improved equations for the prediction of seed longevity. Annals of Botany. 45(1): 13-30.

Eyheraguibel, B., Silvestre, J., \& Morard, P. 2008. Effects of humic substances derived from organic waste enhancement on the growth and mineral nutrition of maize. Bioresource Technology. 99(10): 4206-4212.

Flores, J. D. J., Villegas, A. Y., Castro, R. R., Gómez, V A., Carrillo, R. J., \& Castañeda, H. E. 2020. Inoculación de avena forrajera con hongos micorrízicos arbusculares. Revista Mexicana de Ciencias. 24:191-199.

Flores, G. J. E. 2017. Análisis económico de la producción de pepino (Cucumis sativus L.) híbrido thunder, en el centro de prácticas Manglaralto provincia de Santa Elena. Tesis de Grado. Universidad Estatal Península de Santa Elena. La Libertad (Ecuador).

García, G. J., \& Ocampo, J.A. 2002. Regulation of the plant defence response in arbuscular mycorrhizal symbiosis. Journal of Experimental Botany. 53(373):1377-1386.

González, L.G., Paz, I., Martínez, B., Jiménez, M.C., Torres, J.A., \& Falcón, A. 2015. Respuesta agronómica del cultivo del tomate (Solanum lycopersicum, L) var. HA 3019 a la aplicación de quitosana. UTCiencia Ciencia y Tecnología al servicio del pueblo. 2(2): 55-60.

Leiva, V. D. M. 2018. Eficacia de biofungicidas frente a la caída de plántula de pepino, inducida por Pythium aphanidermatum. Revista de Investigación de Agroproducción Sustentable. 2(1): $72-78$.

López, E. J., Huez L. M., Pacheco, A. F., Jiménez, L. J., \& Preciado F. F. 2011. Productividad y calidad de dos cultivares de pepino en respuesta a la densidad de plantación. Biotecnia. 13(1): 23-28.

Maguire, J.D. 1962. Velocidad de germinación: ayuda a la selección y evaluación de la emergencia y el vigor de las plántulas. Crop Science. 2(2): 176-177.

Medina, G., L.R. 2016. La agricultura, la salinidad y los hongos micorrízicos arbusculares $\bigotimes$ una necesidad, un problema y una alternativa. Cultivos Tropicales. 37(3): 42-49.

Moreno, R.A., García, M. V., Reyes, C. J., Vásquez, A. J., \& Cano, R.P. 2018. Rizobacterias promotoras del crecimiento vegetal: una alternativa de biofertilización para la agricultura sustentable. Rev. Colomb. Biotecnol. 20(1):68-83.
Monirul, I., Humayun, KM, ANK, M. \& Pronabananda, D. 2018. Estudios sobre rendimiento y atributos de rendimiento en tomate y chile mediante aplicación foliar de oligoquitosano. Biological and Pharmaceutical sciencies. 3(3):20-28.

Mujica, P., Y., \& Fuentes, M., A.G. 2012. Efecto a la biofertilización con hongos micorrízicos arbusculares (HMA) en el cultivo del tomate en condiciones de estrés abiótico. Cultivos Tropicales. 33(4): 40-46.

Muscolo, A., Sidari, M., \& Nardi, S. 2013. Sustancia húmica: Relación entre estructura y actividad. La información más profunda sugiere hallazgos unívocos. Revista de exploración geoquímica. 129:57-63.

Pichyangkura, R., \& Chadchawan, S. 2015. Biostimulant activity of chitosan in horticulture. Scientia Horticulturae. 196: 4965.

Ramírez, G. M., Peñaranda, R. A., Pérez M. U., \& Paola S. D. 2018. Biofertilización con hongos formadores de micorrizas arbusculares (HFMA) en especies forestales en vivero. Biotecnología en el Sector Agropecuario y Agroindustrial. 16(2):15-25.

Reyes, P. J. J., Luna, M. R., Reyes, B. M., Yépez, R. Á., Abasolo, P. F., Espinosa, C. K., López, B. R., Vázquez, M. V., Zambrano, B. D., Cabrera, B. D., \& Torres, R. J. 2017. Uso del humus de lombriz y jacinto de agua sobre el crecimiento y desarrollo del pepino (Cucumis sativus, L). Biotecnia. 19 (2): 30-35.

Reyes, P. J. J., Rivero, H.M., García, B. E. L., Beltrán, M. F. A., \& Ruiz, E. F. H. 2020. Aplicación de quitosano incrementa la emergencia, crecimiento y rendimiento del cutivo de tomate (Solanum lycopersicum L.) en condiciones de invernadero. Biotecnia. 22(3): 156-163.

Rivera, R., Fernández, F., Hernández, A., Martin, J., \& Fernández, K. 2003. El manejo efectivo de la simbiosis micorrízica, una vía hacia la agricultura sostenible. Estudio. Cuba: INCA.

Sabreen, K.H., Ibraheim, A., \& Mohsen, A.A. 2015. Effect of Chitosan and nitrogen rates on growth and productivity of summer squash plants. Middle East Journal of Agriculture. 4(4):673-681.

Shehata, S., Fawzy, Z., \& El-Ramady H. 2012. Respuesta de las plantas de pepino a la aplicación foliar de quitosano y levadura en condiciones de invernadero. Revista australiana de ciencias básicas y aplicadas. 4: 63-71.

SIPA. 2018. Sistema de Información Publica Agropecuaria. Quito, Ecuador

Sheikha, S.A., \& Al-Malki, F.M. 2011. Growth and chlorophyll responses of bean plants to the chitosan applications. European Journal of Scientific Research. 50(1): 124-134.

StatSoft Inc. 2018. Statistica. System reference. StatSoft, Inc., Tulsa, Oklahoma, EUA.

Torres, R. J.A., Reyes, P.J.J., González, G., L.G., Jiménez, Pizarro, M., Boicet, F.T., Enríquez, A.E.A., \& González, R.J.C. 2018. Respuesta agronómica de dos variedades de maíz blanco (Zeas mays, L.) a la aplicación de QuitoMax, Azofert y Ecomic. Biotecnia. 20(1):3-7.

Urgiles, G. N., Lalangui ,Z. C., Chamba, Q. E., Loján, P. A., Poma, L. L., Encalada, C. M., \& Aguirre, M. N. 2019. Aislamiento y caracterización morfológica de Hongos Micorrízicos Arbusculares (HMA) de zonas riparias del Sur del Ecuador: un enfoque a la producción de biofertilizantes. Revista del Centro de Estudio y Desarrollo de la Amazonia. 9(1):1-7.

Veobides, A. H., Guridi I. F., \& Vázquez, P. V. 2018. Las sustancias húmicas como bioestimulantes de plantas bajo condiciones de estrés ambiental. Cultivos Tropicales. 39(4): 102-109.

Vital, V. I., Quiñones, A.E., Hernández, M. L., \& Rincón, E.G. 2018. Viabilidad de esporas de hongos micorrízicos arbusculares y semillas de girasol para el establecimiento de la simbiosis micorrízica. Biotecnología y Sustentabilidad. 3(2): 15-25. 